\title{
On complete convergence for weighted sums of martingale-difference random fields
}

Mi Hwa Ko*

"Correspondence:

songhack@wonkwang.ac.kr

Division of Mathematics and

Informational Statistics, Wonkwang

University, Jeonbuk, 570-749, Korea

\begin{abstract}
Let $\left\{a_{\mathbf{n}, \mathbf{i}}, \mathbf{n} \in Z_{+}^{d} \mathbf{i} \leq \mathbf{n}\right\}$ be an array of real numbers, and let $\left\{X_{\mathbf{i}}, \mathbf{i} \in Z_{+}^{d}\right\}$ be the martingale differences with respect to $\left\{\mathcal{G}_{\mathbf{n}}, \mathbf{n} \in Z_{+}^{d}\right\}$ satisfying

$E\left(E\left(X \mid \mathcal{G}_{k}\right) \mid \mathcal{G}_{\mathbf{m}}\right)=E\left(X \mid \mathcal{G}_{\mathbf{k} \wedge \mathbf{m}}\right)$ a.s., where $\mathbf{k} \wedge \mathbf{m}$ denotes componentwise minimum,

$\left\{\mathcal{G}_{\mathbf{k}}, \mathbf{k} \in Z_{+}^{d}\right\}$ is a family of $\sigma$-algebras such that $\forall \mathbf{k} \leq \mathbf{n}, \mathcal{G}_{\mathbf{k}} \subset \mathcal{G}_{\mathbf{n}} \subset \mathcal{G}$, and $X$ is any integrable random variable defined on the initial probability space. The aim of this paper is to obtain some results concerning complete convergence of weighted sums $\sum_{\mathbf{i} \leq \mathbf{n}} a_{\mathbf{n}, \mathbf{i}} X_{\mathbf{i}}$

MSC: $60 \mathrm{~F} 05 ; 60 \mathrm{~F} 15$
\end{abstract}

Keywords: complete convergence; weighted sums; martingale difference; maximal moment inequality; $\sigma$-algebra

\section{Introduction}

The concept of complete convergence for sums of independent and identically distributed random variables was introduced by Hsu and Robbins [1] as follows: A sequence of random variables $\left\{X_{n}\right\}$ is said to be completely to a constant $c$ if

$$
\sum_{n=1}^{\infty} P\left(\left|X_{n}-c\right|>\epsilon\right)<\infty \quad \text { for all } \epsilon>0
$$

This result has been generalized and extended to the random fields $\left\{X_{\mathbf{n}}, \mathbf{n} \in Z_{+}^{d}\right\}$ of random variables. For example, Fazekas and Tómács [2] and Czerebak-Mrozowicz et al. [3] for fields of pairwise independent random variables, and Gut and Stadtmüller [4] for random fields of i.i.d. random variables.

Let $Z_{+}$be the set of positive integers. For fixed $d \in Z_{+}$, set $Z_{+}^{d}=\left\{\mathbf{n}=\left(n_{1}, n_{2}, \ldots, n_{d}\right): n_{i} \in\right.$ $\left.Z_{+}, i=1,2, \ldots, d\right\}$ with coordinatewise partial order, $\leq$, i.e., for $\mathbf{m}=\left(m_{1}, m_{2}, \ldots, m_{d}\right), \mathbf{n}=$ $\left(n_{1}, n_{2}, \ldots, n_{d}\right) \in Z_{+}^{d}, \mathbf{m} \leq \mathbf{n}$ if and only if $m_{i} \leq n_{i}, i=1,2, \ldots, d$. For $\mathbf{n}=\left(n_{1}, n_{2}, \ldots, n_{d}\right) \in Z_{+}^{d}$, let $|\mathbf{n}|=\prod_{i=1}^{d} n_{i}$. For a field $\left\{a_{\mathbf{n}}, \mathbf{n} \in Z_{+}^{d}\right\}$ of real numbers, the limit superior is defined by $\inf _{r \geq 1} \sup _{|\mathbf{n}| \geq r} a_{\mathbf{n}}$ and is denoted by $\lim \sup _{|\mathbf{n}| \rightarrow \infty} a_{\mathbf{n}}$.

Note that $|\mathbf{n}| \rightarrow \infty$ is equivalent to $\max \left\{n_{1}, n_{2}, \ldots, n_{d}\right\} \rightarrow \infty$, which is weaker than the condition $\min \left\{n_{1}, n_{2}, \ldots, n_{d}\right\} \rightarrow \infty$ when $d \geq 2$.

Let $\left\{X_{\mathbf{n}}, \mathbf{n} \in Z_{+}^{d}\right\}$ be a field of random variables, and let $\left\{a_{\mathbf{n}, \mathbf{k}}, \mathbf{n} \in Z_{+}^{d}, \mathbf{k} \leq \mathbf{n}\right\}$ be an array of real numbers. The weighted sums $\sum_{\mathbf{k}<\mathbf{n}} a_{\mathbf{n}, \mathbf{k}} X_{\mathbf{k}}$ can play an important role in various applied and theoretical problems, such as those of the least squares estimators (see Kafles

\section{贷 Springer}

○2013 Ko; licensee Springer. This is an Open Access article distributed under the terms of the Creative Commons Attribution License (http://creativecommons.org/licenses/by/2.0), which permits unrestricted use, distribution, and reproduction in any medium, provided the original work is properly cited. 
and Bhaskara Rao [5]) and $M$-estimates (see Rao and Zhao [6]) in linear models, the nonparametric regression estimators (see Priestley and Chao [7]), etc. So, the study of the limiting behavior of the weighted sums is very important and significant (see Chen and Hao [8]).

Now, we consider the notion of martingale differences. Let $\left\{\mathcal{G}_{\mathbf{k}}, \mathbf{k} \in Z_{+}^{d}\right\}$ be a family of $\sigma$-algebras such that

$$
\mathcal{G}_{\mathbf{k}} \subset \mathcal{G}_{\mathbf{n}} \subset \mathcal{G}, \quad \forall \mathbf{k} \leq \mathbf{n}
$$

and for any integrable random variable $X$ defined on the initial probability space,

$$
E\left(E\left(X \mid \mathcal{G}_{\mathbf{k}}\right) \mid \mathcal{G}_{\mathbf{m}}\right)=E\left(X \mid \mathcal{G}_{\mathbf{k} \wedge \mathbf{m}}\right) \quad \text { a.s. }
$$

where $\mathbf{k} \wedge \mathbf{m}$ denotes the componentwise minimum.

An $\left\{\mathcal{G}_{\mathbf{k}}, \mathbf{k} \in Z_{+}^{d}\right\}$-adapted, integrable process $\left\{Y_{\mathbf{k}}, \mathbf{k} \in Z_{+}^{d}\right\}$ is called a martingale if and only if

$$
E\left(Y_{\mathbf{n}} \mid \mathcal{G}_{\mathbf{m}}\right)=Y_{\mathbf{m} \wedge \mathbf{n}} \quad \text { a.s. }
$$

Let us observe that for martingale $\left\{\left(Y_{\mathbf{n}}, \mathcal{G}_{\mathbf{n}}\right), \mathbf{n} \in Z_{+}^{d}\right\}$, the random variables

$$
X_{\mathbf{n}}=\sum_{\mathbf{a} \in\{0,1\}^{d}}(-1)^{\sum_{\mathbf{i}=1}^{d} a_{i}} Y_{\mathbf{n}-\mathbf{a}},
$$

where $\mathbf{a}=\left(a_{1}, a_{2}, \ldots, a_{d}\right)$ and $\mathbf{n} \in Z_{+}^{d}$, are martingale differences with respect to $\left\{\mathcal{G}_{\mathbf{n}}, \mathbf{n} \in Z_{+}^{d}\right\}$ (see Kuczmaszewska and Lagodowski [9]).

For the results concerning complete convergence for martingale arrays obtained in the one-dimensional case, we refer to Lagodowski and Rychlik [10], Elton [11], Lesigne and Volny [12], Stoica [13] and Ghosal and Chandra [14]. Recently, complete convergence for martingale difference random fields was proved by Kuczmaszewska and Lagodowski [9].

The aim of this paper is to obtain some results concerning complete convergence of weighted sums $\sum_{\mathbf{i} \leq \mathbf{n}} a_{\mathbf{n}, \mathbf{i}} X_{\mathbf{i}}$, where $\left\{a_{\mathbf{n}, \mathbf{i}}, \mathbf{n} \in Z_{+}^{d}, \mathbf{i} \leq \mathbf{n}\right\}$ is an array of real numbers, and $\left\{X_{\mathbf{i}}, \mathbf{i} \in Z_{+}^{d}\right\}$ is the martingale differences with respect to $\left\{\mathcal{G}_{\mathbf{n}}, \mathbf{n} \in Z_{+}^{d}\right\}$ satisfying (1.1).

\section{Results}

The following moment maximal inequality provides us a useful tool to prove the main results of this section (see Kuczmaszewska and Lagodowski [9]).

Lemma 2.1 Let $\left\{\left(Y_{\mathbf{n}}, \mathcal{G}_{\mathbf{n}}\right), \mathbf{n} \in Z_{+}^{d}\right\}$ be a martingale, and let $\left\{\left(X_{\mathbf{n}}, \mathcal{G}_{\mathbf{n}}\right), \mathbf{n} \in Z_{+}^{d}\right\}$ be the martingale differences corresponding to it. Let $q>1$. There exists a finite and positive constant $C$ depending only on $q$ and $d$ such that

$$
E\left(\max _{\mathbf{k} \leq \mathbf{n}}\left|Y_{\mathbf{k}}\right|^{q}\right) \leq C E\left(\sum_{\mathbf{k} \leq \mathbf{n}} X_{\mathbf{k}}^{2}\right)^{q / 2} .
$$

Let us denote $\mathcal{G}_{\mathbf{i}}^{*}=\sigma\left\{\mathcal{G}_{\mathbf{j}}: \mathbf{j}<\mathbf{i}\right\}$. Now, we are ready to formulate the next result. 
Theorem 2.2 Let $\left\{a_{\mathbf{n}}, \mathbf{i}, \mathbf{n} \in Z_{+}^{d}, \mathbf{i} \leq \mathbf{n}\right\}$ be an array of real numbers, and let $\left\{X_{\mathbf{n}}, \mathbf{n} \in Z_{+}^{d}\right\}$ be the martingale differences with respect to $\left\{\mathcal{G}_{\mathbf{n}}, \mathbf{n} \in Z_{+}^{d}\right\}$ satisfying (1.1). For $\alpha p>1, p>1$ and $\alpha>\frac{1}{2}$, we assume that

(i) $\sum_{\mathbf{n}}|\mathbf{n}|^{\alpha p-2} \sum_{\mathbf{i} \leq \mathbf{n}} P\left\{\left|a_{\mathbf{n}, \mathbf{i}} X_{\mathbf{i}}\right|>|\mathbf{n}|^{\alpha}\right\}<\infty$,

(ii) $\sum_{\mathbf{n}}|\mathbf{n}|^{\alpha(p-q)-3+q / 2} \sum_{\mathbf{i} \leq \mathbf{n}}\left|a_{\mathbf{n}, \mathbf{i}}\right|^{q} E\left(\left|X_{\mathbf{i}}\right|^{q} I\left[\left|a_{\mathbf{n}, \mathbf{i}} X_{\mathbf{i}}\right| \leq|\mathbf{n}|^{\alpha}\right]\right)<\infty$ for $q \geq 2$,

(ii) ${ }^{\prime} \sum_{\mathbf{n}}|\mathbf{n}|^{\alpha(p-q)-2} \sum_{\mathbf{i} \leq \mathbf{n}}\left|a_{\mathbf{n}, \mathbf{i}}\right|^{q} E\left(\left|X_{\mathbf{i}}\right|^{q} I\left[\left|a_{\mathbf{n}, \mathbf{i}} X_{\mathbf{i}}\right| \leq|\mathbf{n}|^{\alpha}\right]\right)<\infty$ for $1<q<2$ and

(iii) $\sum_{\mathbf{n}}|\mathbf{n}|^{\alpha p-2} P\left\{\max _{\mathbf{j} \leq \mathbf{n}}\left|\sum_{\mathbf{i} \leq \mathbf{j}} E\left(a_{\mathbf{n}, \mathbf{i}} X_{\mathbf{i}} I\left[\left|a_{\mathbf{n}, \mathbf{i}} X_{\mathbf{i}}\right| \leq|\mathbf{n}|^{\alpha}\right] \mid \mathcal{G}_{\mathbf{i}}^{*}\right)\right|>\epsilon|\mathbf{n}|^{\alpha}\right\}<\infty$ for all $\epsilon>0$.

Then we have

$$
\sum_{\mathbf{n}}|\mathbf{n}|^{\alpha p-2} P\left\{\max _{\mathbf{j} \leq \mathbf{n}}\left|S_{\mathbf{j}}\right|>\epsilon|\mathbf{n}|^{\alpha}\right\}<\infty \quad \text { for all } \epsilon>0
$$

where $S_{\mathbf{n}}=\sum_{\mathbf{1} \leq \mathbf{i} \leq \mathbf{n}} a_{\mathbf{n}, \mathbf{i}} X_{\mathbf{i}}$

Proof Let us notice that the series $\sum_{\mathbf{n}}|\mathbf{n}|^{\alpha p-2}$ is finite, then (2.2) always holds. Therefore, we consider only the case such that $\sum_{\mathbf{n}}|\mathbf{n}|^{\alpha p-2}$ is divergent. Let $X_{\mathbf{n}, \mathbf{i}}=X_{\mathbf{i}} I\left[\left|a_{\mathbf{n}, \mathbf{i}} X_{\mathbf{i}}\right| \leq|\mathbf{n}|^{\alpha}\right]$, $X_{\mathbf{n}, \mathbf{i}}^{*}=X_{\mathbf{n}, \mathbf{i}}-E\left(X_{\mathbf{n}, \mathbf{i}} \mid \mathcal{G}_{\mathbf{i}}^{*}\right)$ and $S_{\mathbf{n}, \mathbf{j}}^{*}=\sum_{\mathbf{i} \leq \mathbf{j}} a_{\mathbf{n}, \mathbf{i}} X_{\mathbf{n}, \mathbf{i}}^{*}$

Then

$$
\begin{aligned}
\sum_{\mathbf{n}}|\mathbf{n}|^{\alpha p-2} P\left\{\max _{\mathbf{j} \leq \mathbf{n}}\left|S_{\mathbf{j}}\right|>\epsilon|\mathbf{n}|^{\alpha}\right\} \\
\leq \sum_{\mathbf{n}}|\mathbf{n}|^{\alpha p-2} P\left\{\left|a_{\mathbf{n}, \mathbf{i}} X_{\mathbf{i}}\right|>|\mathbf{n}|^{\alpha}\right\} \\
\quad+\sum_{\mathbf{n}}|\mathbf{n}|^{\alpha p-2} P\left\{\max _{\mathbf{j} \leq \mathbf{n}}\left|\sum_{\mathbf{i} \leq \mathbf{j}} a_{\mathbf{n}, \mathbf{i}} X_{\mathbf{i}} I\left[\left|a_{\mathbf{n}, \mathbf{i}} X_{\mathbf{i}}\right| \leq|\mathbf{n}|^{\alpha}\right]\right|>\epsilon|\mathbf{n}|^{\alpha}\right\} \\
\leq \sum_{\mathbf{n}}|\mathbf{n}|^{\alpha p-2} \sum_{\mathbf{i} \leq \mathbf{n}} P\left\{\left|a_{\mathbf{n}, \mathbf{i}} X_{\mathbf{i}}\right|>|\mathbf{n}|^{\alpha}\right\}+\sum_{\mathbf{n}}|\mathbf{n}|^{\alpha p-2} P\left\{\max _{\mathbf{j} \leq \mathbf{n}} \mid \sum_{\mathbf{i} \leq \mathbf{j}}\left(a_{\mathbf{n}, \mathbf{i}} X_{\mathbf{i}} I\left[\left|a_{\mathbf{n}, \mathbf{i}} X_{\mathbf{i}}\right| \leq|\mathbf{n}|^{\alpha}\right]\right.\right. \\
\left.\left.\quad-E\left(a_{\mathbf{n}, \mathbf{i}} X_{\mathbf{i}} I\left[\left|a_{\mathbf{n}, \mathbf{i}} X_{\mathbf{i}}\right| \leq|\mathbf{n}|^{\alpha}\right] \mid \mathcal{G}_{\mathbf{i}}^{*}\right)\right)\left.\left|>\frac{\epsilon}{2}\right| \mathbf{n}\right|^{\alpha}\right\} \\
\quad+\sum_{\mathbf{n}}|\mathbf{n}|^{\alpha p-2} P\left\{\max _{\mathbf{j} \leq \mathbf{n}}\left|\sum_{\mathbf{i} \leq \mathbf{j}} E\left(a_{\mathbf{n}, \mathbf{i}} X_{\mathbf{i}} I\left[\left|a_{\mathbf{n}, \mathbf{i}} X_{\mathbf{i}}\right| \leq|\mathbf{n}|^{\alpha}\right] \mid \mathcal{G}_{\mathbf{i}}^{*}\right)\right|>\frac{\epsilon}{2}|\mathbf{n}|^{\alpha}\right\} \\
=I_{1}+I_{2}+I_{3} .
\end{aligned}
$$

Clearly, $I_{1}<\infty$ by (i), and $I_{3}<\infty$ by (iii). It remains to prove that $I_{2}<\infty$. Thus, the proof will be completed by proving that

$$
\sum_{\mathbf{n}}|\mathbf{n}|^{\alpha p-2} P\left\{\max _{\mathbf{j} \leq \mathbf{n}}\left|S_{\mathbf{n}, \mathbf{j}}^{*}\right|>\epsilon|\mathbf{n}|^{\alpha}\right\}<\infty .
$$

To prove it, we first observe that $\left\{\left(S_{\mathbf{n}, \mathbf{j}}^{*}, \mathcal{G}_{\mathbf{j}}\right), \mathbf{j} \leq \mathbf{n}\right\}$ is a martingale. In fact, if $\mathbf{i}>\mathbf{j}$, then $\mathcal{G}_{\mathbf{i} \wedge \mathbf{j}} \subset$ $\mathcal{G}_{\mathbf{i}}^{*}$ and by (1.1), we have

$$
\begin{aligned}
E\left(a_{\mathbf{n}, \mathbf{i}} X_{\mathbf{n}, \mathbf{i}}^{*} \mid \mathcal{G}_{\mathbf{j}}\right) & =E\left(a_{\mathbf{n}, \mathbf{i}} X_{\mathbf{n}, \mathbf{i}}-E\left(a_{\mathbf{n}, \mathbf{i}} X_{\mathbf{n}, \mathbf{i}} \mid \mathcal{G}_{\mathbf{i}}^{*}\right) \mid \mathcal{G}_{\mathbf{i}}\right) \\
& =E\left(E\left(a_{\mathbf{n}, \mathbf{i}} X_{\mathbf{n}, \mathbf{i}}-E\left(a_{\mathbf{n}, \mathbf{i}} X_{\mathbf{n}, \mathbf{i}} \mid \mathcal{G}_{\mathbf{i}}^{*}\right) \mid \mathcal{G}_{\mathbf{i}}\right) \mid \mathcal{G}_{\mathbf{j}}\right)
\end{aligned}
$$




$$
\begin{aligned}
& =E\left(a_{\mathbf{n}, \mathbf{i}} X_{\mathbf{n}, \mathbf{i}}-E\left(a_{\mathbf{n}, \mathbf{i}} X_{\mathbf{n}, \mathbf{i}} \mid \mathcal{G}_{\mathbf{i}}^{*}\right) \mid \mathcal{G}_{\mathbf{i} \wedge \mathbf{j}}\right) \\
& =0
\end{aligned}
$$

Then, by the Markov inequality and Lemma 2.1, there exists some constant $C$ such that

$$
\begin{aligned}
P\left\{\max _{\mathbf{j} \leq \mathbf{n}}\left|S_{\mathbf{n}, \mathbf{j}}^{*}\right|>\epsilon|\mathbf{n}|^{\alpha}\right\} & \leq C \frac{E\left(\max _{\mathbf{j} \leq \mathbf{n}}\left|S_{\mathbf{n}, \mathbf{j}}^{*}\right|^{q}\right)}{|\mathbf{n}|^{q q}} \\
& \leq \frac{C}{|\mathbf{n}|^{\alpha q}} E\left(\sum_{\mathbf{i} \leq \mathbf{n}} a_{\mathbf{n}, \mathbf{i}}^{2} X_{\mathbf{n}, \mathbf{i}}^{* 2}\right)^{q / 2}=I_{4} .
\end{aligned}
$$

Case $q \geq 2$; we get

$$
\begin{aligned}
I_{4} & \leq \frac{C}{|\mathbf{n}|^{\alpha q}}|\mathbf{n}|^{q / 2-1} \sum_{\mathbf{i} \leq \mathbf{n}} E\left|a_{\mathbf{n}, \mathbf{i}} X_{\mathbf{n}, \mathbf{i}}^{*}\right|^{q} \\
& \leq C|\mathbf{n}|^{q / 2-1-\alpha q} \sum_{\mathbf{i} \leq \mathbf{n}} E\left(\left|a_{\mathbf{n}, \mathbf{i}} X_{\mathbf{i}}\right|^{q} I\left[\left|a_{\mathbf{n}, \mathbf{i}} X_{\mathbf{i}}\right| \leq|\mathbf{n}|^{\alpha}\right]\right) .
\end{aligned}
$$

Note that the last estimation follows from the Jensen inequality. Thus, we have

$$
\begin{aligned}
& \sum_{\mathbf{n}}|\mathbf{n}|^{\alpha p-2} P\left\{\max _{\mathbf{j} \leq \mathbf{n}}\left|S_{\mathbf{n}, \mathbf{j}}^{*}\right|>\epsilon|\mathbf{n}|^{\alpha}\right\} \\
& \quad \leq C \sum_{\mathbf{n}}|\mathbf{n}|^{\alpha p-3-q(\alpha-1 / 2)} \sum_{\mathbf{i} \leq \mathbf{n}} E\left(\left|a_{\mathbf{n}, \mathbf{i}} X_{\mathbf{i}}\right|^{q} I\left[\left|a_{\mathbf{n}, \mathbf{i}} X_{\mathbf{i}}\right| \leq|\mathbf{n}|^{\alpha}\right]\right)<\infty
\end{aligned}
$$

by assumption (ii).

Case $1<q<2$; we get

$$
\begin{aligned}
I_{4} & \leq \frac{C}{|\mathbf{n}|^{\alpha q}} \sum_{\mathbf{i} \leq \mathbf{n}} E\left|a_{\mathbf{n}, \mathbf{i}} X_{\mathbf{n}, \mathbf{i}}^{*}\right|^{q} \\
& \leq C|\mathbf{n}|^{-\alpha q} \sum_{\mathbf{i} \leq \mathbf{n}} E\left(\left|a_{\mathbf{n}, \mathbf{i}} X_{\mathbf{i}}\right|^{q} I\left[\left|a_{\mathbf{n}, \mathbf{i}} X_{\mathbf{i}}\right| \leq|\mathbf{n}|^{\alpha}\right]\right) .
\end{aligned}
$$

Therefore, for $1<q<2$, we obtain

$$
\begin{aligned}
& \sum_{\mathbf{n}}|\mathbf{n}|^{\alpha p-2} P\left\{\max _{\mathbf{j} \leq \mathbf{n}}\left|S_{\mathbf{n}, \mathbf{j}}^{*}\right|>\epsilon|\mathbf{n}|^{\alpha}\right\} \\
& \quad \leq C \sum_{\mathbf{n}}|\mathbf{n}|^{\alpha(p-q)-2} \sum_{\mathbf{i} \leq \mathbf{n}} E\left(\left|a_{\mathbf{n}, \mathbf{i}} X_{\mathbf{i}}\right|^{q} I\left[\left|a_{\mathbf{n}, \mathbf{i}} X_{\mathbf{i}}\right| \leq|\mathbf{n}|^{\alpha}\right]\right)<\infty
\end{aligned}
$$

by assumption (ii)'. Thus, $I_{2}<\infty$ for all $q>1$, and the proof of Theorem 2.2 is complete.

Corollary 2.3 Let $\left\{a_{\mathbf{n}}, \mathbf{i}, \mathbf{n} \in Z_{+}^{d}, \mathbf{i} \leq \mathbf{n}\right\}$ be an array of real numbers. Let $\left\{X_{\mathbf{n}}, \mathbf{n} \in Z_{+}^{d}\right\}$ be martingale differences with respect to $\left\{\mathcal{G}_{\mathbf{n}}, \mathbf{n} \in Z_{+}^{d}\right\}$ satisfying (1.1), and $E X_{\mathbf{n}}=0$ for $\mathbf{n} \in Z_{+}^{d}$. Let $p \geq 1, \alpha>\frac{1}{2}$ and $\alpha p>1$. Assume that (i) and for some $q>1$, (ii) or (ii)' hold respectively. If

$$
\max _{\mathbf{j} \leq \mathbf{n}} \sum_{\mathbf{i} \leq \mathbf{j}} E\left(a_{\mathbf{n}, \mathbf{i}} X_{\mathbf{i}} I\left[\left|a_{\mathbf{n}, \mathbf{i}} X_{\mathbf{i}}\right| \leq|\mathbf{n}|^{\alpha}\right] \mid \mathcal{G}_{\mathbf{i}}^{*}\right)=o\left(|\mathbf{n}|^{\alpha}\right),
$$

then (2.2) holds. 
Proof It is easy to see that (2.3) implies (iii). We omit details that prove it.

The following corollary shows that assumption (iii) in Theorem 2.2 is natural, and in the case of independent random fields, it reduces to the known one.

Corollary 2.4 Let $\left\{a_{\mathbf{n}}, \mathbf{i}, \mathbf{n} \in Z_{+}^{d}, \mathbf{i} \leq \mathbf{n}\right\}$ be an array of real numbers. Let $\left\{X_{\mathbf{n}}, \mathbf{n} \in Z_{+}^{d}\right\}$ be a field of independent random variables such that $E X_{\mathbf{n}}=0$ for $\mathbf{n} \in Z_{+}^{d}$. Let $p \geq 1, \alpha>\frac{1}{2}$ and $\alpha p>1$. Assume that (i) and for some $q>1$, (ii) or (ii)' hold respectively. If

$$
\frac{1}{|\mathbf{n}|^{\alpha}} \max _{\mathbf{j} \leq \mathbf{n}} \sum_{\mathbf{i} \leq \mathbf{j}} E\left(a_{\mathbf{n}, \mathbf{i}} X_{\mathbf{i}} I\left[\left|a_{\mathbf{n}, \mathbf{i}} X_{\mathbf{i}}\right| \leq|\mathbf{n}|^{\alpha}\right]\right) \rightarrow 0 \quad \text { as }|\mathbf{n}| \rightarrow \infty,
$$

then (2.2) holds.

Proof Since $\left\{X_{\mathbf{n}}, \mathbf{n} \in Z_{+}^{d}\right\}$ is a field of independent random variables, we have

$$
\frac{1}{|\mathbf{n}|^{\alpha}} \max _{\mathbf{j} \leq \mathbf{n}} \sum_{\mathbf{i} \leq \mathbf{j}} E\left(a_{\mathbf{n}, \mathbf{i}} X_{\mathbf{i}} I\left[\left|a_{\mathbf{n}, \mathbf{i}} X_{\mathbf{i}}\right| \leq|\mathbf{n}|^{\alpha}\right] \mid \mathcal{G}_{\mathbf{i}}^{*}\right)=\frac{1}{|\mathbf{n}|^{\alpha}} \max _{\mathbf{j} \leq \mathbf{n}} \sum_{\mathbf{i} \leq \mathbf{j}} E\left(a_{\mathbf{n}, \mathbf{i}} X_{\mathbf{i}} I\left[\left|a_{\mathbf{n}, \mathbf{i}} X_{\mathbf{i}}\right| \leq|\mathbf{n}|^{\alpha}\right]\right) .
$$

Now, it is easy to see that (2.4) implies (iii) of Theorem 2.2. Thus, by Theorem 2.2, result (2.2) follows.

Remark Theorem 2.2 and Corollary 2.4 are extensions of Theorem 4.1 and Corollary 4.1 in Kuczmaszewska and Lagodowski [9] to the weighted sums case, respectively.

Corollary 2.5 Let $\left\{a_{\mathbf{n}}, \mathbf{i}, \mathbf{n} \in Z_{+}^{d}, \mathbf{1} \leq \mathbf{i} \leq \mathbf{n}\right\}$ be an array of real numbers. Let $\left\{X_{\mathbf{n}}, \mathbf{n} \in Z_{+}^{d}\right\}$ be the martingale differences with respect to $\left\{\mathcal{G}_{\mathbf{n}}, \mathbf{n} \in Z_{+}^{d}\right\}$ satisfying (1.1) and $E X_{\mathbf{n}}=0$. Let $p \geq 1, \alpha>\frac{1}{2}$ and $\alpha p>1$ and $E\left|X_{\mathbf{n}}\right|^{1+\lambda_{\mathbf{n}}}<\infty$ for $\lambda_{\mathbf{n}}$ with $0<\lambda_{\mathbf{n}}<1$ for $\mathbf{n} \in Z_{+}^{d}$. If

$$
\begin{aligned}
& \sum_{\mathbf{n}}|\mathbf{n}|^{\alpha p-2}|\mathbf{n}|^{-\alpha\left(1+\lambda_{\mathbf{n}}\right)} \sum_{\mathbf{i} \leq \mathbf{n}}\left|a_{\mathbf{n}, \mathbf{i}}\right|^{1+\lambda_{\mathbf{n}}} E\left|X_{\mathbf{i}}\right|^{1+\lambda_{\mathbf{n}}}<\infty, \\
& \max _{\mathbf{1} \leq \mathbf{j} \leq \mathbf{n}} \sum_{\mathbf{i} \leq \mathbf{j}} E\left(\left|a_{\mathbf{n}, \mathbf{i}} X_{i}\right| I\left[\left|a_{\mathbf{n}, \mathbf{i}} X_{\mathbf{i}}\right| \leq|\mathbf{n}|^{\alpha}\right] \mid \mathcal{G}_{\mathbf{i}}^{*}\right)=o\left(|\mathbf{n}|^{\alpha}\right),
\end{aligned}
$$

then (2.2) holds.

Proof If the series $\sum_{\mathbf{n}}|\mathbf{n}|^{\alpha p-2}<\infty$, then (2.2) always holds. Hence, we only consider the case $\sum_{\mathbf{n}}|\mathbf{n}|^{\alpha p-2}=\infty$. It follows from (2.5) that

$$
|\mathbf{n}|^{-\alpha\left(1+\lambda_{\mathbf{n}}\right)} \sum_{\mathbf{i} \leq \mathbf{n}}\left|a_{\mathbf{n}, \mathbf{i}}\right|^{1+\lambda_{\mathbf{n}}} E\left|X_{i}\right|^{1+\lambda_{\mathbf{n}}}<1 .
$$

By (2.5) and the Markov inequality,

$$
\begin{aligned}
& \sum_{\mathbf{n}}|\mathbf{n}|^{\alpha p-2} P\left(\left|a_{\mathbf{n}, \mathbf{i}} X_{\mathbf{i}}\right|>|\mathbf{n}|^{\alpha}\right) \\
& \quad \leq \sum_{\mathbf{n}}|\mathbf{n}|^{\alpha p-2}|\mathbf{n}|^{-\alpha\left(1+\lambda_{\mathbf{n}}\right)} \sum_{\mathbf{i} \leq \mathbf{n}}\left|a_{\mathbf{n}, \mathbf{i}}\right|^{1+\lambda_{\mathbf{n}}} E\left|X_{\mathbf{i}}\right|^{1+\lambda_{\mathbf{n}}}<\infty,
\end{aligned}
$$

which satisfies (i) of Theorem 2.2. 
As the proof of Corollary 2.3, (2.6) implies (iii) of Theorem 2.2.

It remains to show that Theorem 2.2(ii) or (ii)' is satisfied.

For $1<q<2$, take $1+\lambda_{\mathbf{n}}<q$. Then we have

$$
\begin{aligned}
& \sum_{\mathbf{n}}|\mathbf{n}|^{\alpha(p-q)-2} \sum_{\mathbf{i} \leq \mathbf{n}}\left|a_{\mathbf{n}, \mathbf{i}}\right|^{q} E\left(\left|X_{\mathbf{i}}\right|^{q} I\left[\left|a_{\mathbf{n}, \mathbf{i}} X_{\mathbf{i}}\right| \leq|\mathbf{n}|^{\alpha}\right]\right) \\
& \quad \leq \sum_{\mathbf{n}}|\mathbf{n}|^{\alpha p-2}|\mathbf{n}|^{-\alpha\left(1+\lambda_{\mathbf{n}}\right)}|\mathbf{n}|^{-\alpha q+\alpha\left(1+\lambda_{\mathbf{n}}\right)}|\mathbf{n}|^{\alpha q-\alpha\left(1+\lambda_{\mathbf{n}}\right)} \sum_{\mathbf{i} \leq \mathbf{n}}\left|a_{\mathbf{n}, \mathbf{i}}\right|^{1+\lambda_{\mathbf{n}}} E\left|X_{\mathbf{i}}\right|^{1+\lambda_{\mathbf{n}}} \\
& \quad=\sum_{\mathbf{n}}|\mathbf{n}|^{\alpha p-2}|\mathbf{n}|^{-\alpha\left(1+\lambda_{\mathbf{n}}\right)} \sum_{\mathbf{i} \leq \mathbf{n}}\left|a_{\mathbf{n}, \mathbf{i}}\right|^{1+\lambda_{\mathbf{n}}} E\left|X_{i}\right|^{1+\lambda_{\mathbf{n}}}<\infty \quad \text { by }(2.5),
\end{aligned}
$$

which satisfies Theorem 2.2(ii)'. Hence, the proof is complete.

Corollary 2.6 Let $\left\{a_{\mathbf{n}}, \mathbf{i}, \mathbf{n} \in Z_{+}^{d}, \mathbf{1} \leq \mathbf{i} \leq \mathbf{n}\right\}$ be an array of real numbers, and let $\left\{X_{\mathbf{n}}, \mathbf{n} \in\right.$ $\left.Z_{+}^{d}\right\}$ be the martingale differences with respect to $\left\{\mathcal{G}_{\mathbf{n}}, \mathbf{n} \in Z_{+}^{d}\right\}$ satisfying (1.1), $E X_{\mathbf{n}}=0$ and $E\left|X_{\mathbf{n}}\right|^{p}<\infty$ for $1<p<2$. Let $\alpha>\frac{1}{2}, \alpha p>1$ and $1<p<2$. If

$$
\sum_{\mathbf{1} \leq \mathbf{i} \leq \mathbf{n}}\left|a_{\mathbf{n}, \mathbf{i}}\right|^{p} E\left|X_{\mathbf{i}}\right|^{p}=O\left(|\mathbf{n}|^{\delta}\right) \quad \text { for } 0<\delta<1,
$$

and Theorem 2.2(iii) hold, then (2.2) holds.

Proof By (2.8) and the Markov inequality,

$$
\begin{aligned}
& \sum_{\mathbf{n}}|\mathbf{n}|^{\alpha p-2} \sum_{\mathbf{i} \leq \mathbf{n}} P\left(\left|a_{\mathbf{n}, \mathbf{i}} X_{\mathbf{i}}\right|>|\mathbf{n}|^{\alpha}\right) \\
& \leq \sum_{\mathbf{n}}|\mathbf{n}|^{\alpha p-2} \sum_{\mathbf{i} \leq \mathbf{n}} \frac{\left|a_{\mathbf{n}, \mathbf{i}}\right|^{p} E\left|X_{\mathbf{i}}\right|^{p}}{|\mathbf{n}|^{\alpha p}} \\
& \leq C \sum_{\mathbf{n}}|\mathbf{n}|^{-2+\delta}<\infty .
\end{aligned}
$$

By taking $q<p$, we have

$$
\begin{aligned}
& \sum_{\mathbf{n}}|\mathbf{n}|^{\alpha(p-q)-2} \sum_{\mathbf{i} \leq \mathbf{n}}\left|a_{\mathbf{n}, \mathbf{i}}\right|^{q} E\left(\left|X_{\mathbf{i}}\right|^{q} I\left[\left|a_{\mathbf{n}, \mathbf{i}} X_{\mathbf{i}}\right| \leq \epsilon|\mathbf{n}|^{\alpha}\right]\right) \\
& \leq \sum_{\mathbf{n}}|\mathbf{n}|^{-2} \sum_{\mathbf{i} \leq \mathbf{n}}\left|a_{\mathbf{n}, \mathbf{i}}\right|^{p} E\left|X_{\mathbf{i}}\right|^{p} \\
& \quad \leq C \sum_{\mathbf{n}}|\mathbf{n}|^{-2+\delta}<\infty .
\end{aligned}
$$

Hence, by (2.9) and (2.10), conditions (i) and (ii)' in Theorem 2.2 are satisfied, respectively.

To complete the proof, it is enough to note that by $E X_{\mathbf{n}}=0$ for $\mathbf{n} \in Z_{+}^{d}$ and by (2.8), we get for $\mathbf{j} \leq \mathbf{n}$

$$
|\mathbf{n}|^{-\alpha} \sum_{\mathbf{i} \leq \mathbf{j}}\left|a_{\mathbf{n}, \mathbf{i}}\right| E\left|X_{\mathbf{i}}\right| I\left[\left|a_{\mathbf{n}, \mathbf{i}} X_{\mathbf{i}}\right| \leq \epsilon|\mathbf{n}|^{\alpha}\right] \rightarrow 0 \quad \text { as }|\mathbf{n}| \rightarrow \infty .
$$

Hence, the proof is complete. 
Corollary 2.7 Let $\left\{X_{\mathbf{n}}, \mathbf{n} \in Z_{+}^{d}\right\}$ be the martingale differences with respect to $\left\{\mathcal{G}_{\mathbf{n}}, \mathbf{n} \in Z_{+}^{d}\right\}$ satisfying (1.1), let $E X_{\mathbf{n}}=0$ and $E\left|X_{\mathbf{n}}\right|^{p}<\infty$ for $1<p<2$ and be stochastically dominated by a random variable $X$, i.e., there is a constant $D$ such that $P\left(\left|X_{\mathbf{n}}\right|>x\right) \leq D P(|X|>x)$ for all $x \geq 0$ and $\mathbf{n} \in Z_{+}^{d}$. Let $\left\{a_{\mathbf{n}}, \mathbf{i}, \mathbf{n} \in Z_{+}^{d}, \mathbf{i} \leq \mathbf{n}\right\}$ be an array of real numbers satisfying

$$
\sum_{\mathbf{i} \leq \mathbf{n}}\left|a_{\mathbf{n}, \mathbf{i}}\right|^{p}=O\left(|\mathbf{n}|^{\delta}\right) \quad \text { for } 0<\delta<1 .
$$

If Theorem 2.2(iii) holds, then (2.2) holds.

Proof From (2.12), (2.8) follows. Hence, by Corollary 2.6, we obtain (2.2).

Remark Linear random fields are of great importance in time series analysis. They arise in a wide variety of context. Applications to economics, engineering, and physical science are extremely broad (see Kim et al. [15]).

Let $Y_{\mathbf{k}}=\sum_{\mathbf{i} \geq \mathbf{1}} a_{\mathbf{k}+\mathbf{i}} X_{\mathbf{i}}$, where $\left\{a_{\mathbf{i}}, \mathbf{i} \in Z_{+}^{d}\right\}$ is a field of real numbers with $\sum_{\mathbf{i}} \geq \mathbf{1}\left|a_{\mathbf{i}}\right|<\infty$, and $\left\{X_{\mathbf{i}}, \mathbf{i} \in Z_{+}^{d}\right\}$ is a field of the martingale difference random variables.

Define $a_{\mathbf{n}, \mathbf{i}}=\sum_{\mathbf{1} \leq \mathbf{k} \leq \mathbf{n}} a_{\mathbf{i}+\mathbf{k}}$. Then we have

$$
\sum_{1 \leq \mathbf{k} \leq \mathbf{n}} Y_{\mathbf{k}}=\sum_{1 \leq \mathbf{k} \leq \mathbf{n}} \sum_{\mathbf{i} \geq \mathbf{1}} a_{\mathbf{i}+\mathbf{k}} X_{\mathbf{i}}=\sum_{\mathbf{i} \geq \mathbf{1}} \sum_{\mathbf{1} \leq \mathbf{k} \leq \mathbf{n}} a_{\mathbf{i}+\mathbf{k}} X_{\mathbf{i}}=\sum_{\mathbf{i} \geq \mathbf{1}} a_{\mathbf{n}, \mathbf{i}} X_{\mathbf{i}}
$$

Hence, we can use the above results to investigate the complete convergence for linear random fields.

Competing interests

The author declares that she has no competing interests.

Received: 21 June 2013 Accepted: 23 September 2013 Published: 07 Nov 2013

\section{References}

1. Hsu, PL, Robbins, H: Complete convergence and the law of large numbers. Proc. Natl. Acad. Sci. USA 33, 25-31 (1947)

2. Fazekas, I, Tómács, T: Strong laws of large numbers for pairwise independent random variables with multidimensional indices. Publ. Math. (Debr.) 53, 149-161 (1998)

3. Czerebak-Mrozowicz, EB, Klesov, OI, Rychlik, Z: Marcinkiewicz-type strong law of large numbers for pairwise independent random fields. Probab. Math. Stat. 22, 127-139 (2002)

4. Gut, A, Stadtmüller, U: An asymmetric Marcinkiewicz-Zygmund SLLN for random fields. Stat. Probab. Lett. 35, 756-763 (2009)

5. Kafles, D, Bhaskara Rao, M: Weak consistency of least squares estimators in linear models. J. Multivar. Anal. 12, 186-198 (1982)

6. Rao, CR, Zhao, LC: Linear representation of M-estimates in linear models. Can. J. Stat. 20, 359-368 (1992)

7. Priestley, MB, Chao, MT: Nonparametric function fitting. J. R. Stat. Soc. B 34, 385-392 (1972)

8. Chen, $\mathrm{P}, \mathrm{Hao}, \mathrm{C}$ : A remark on the law of the logarithm for weighted sums of random variables with multidimensional indices. Stat. Probab. Lett. 81, 1808-1812 (2011)

9. Kuczmaszewska, A, Lagodowski, Z: Convergence rates in the SLLN for some classes of dependent random fields. J. Math. Anal. Appl. 380, 571-584 (2011)

10. Lagodowski, ZA, Rychlik, Z: Rate of convergence in the strong law of large numbers for martingales. Probab. Theory Relat. Fields 71, 467-476 (1986)

11. Elton, J: Law of large numbers for identically distributed martingale differences. Ann. Probab. 9, 405-412 (1981)

12. Lesigne, E, Volny, D: Large deviations for martingales. Stoch. Process. Appl. 96, 143-159 (2001)

13. Stoica, G: Baum-Katz-Nagayev type results for martingales. J. Math. Anal. Appl. 336, 1489-1492 (2007)

14. Ghosal, S, Chandra, TK: Complete convergence of martingale arrays. J. Theor. Probab. 11, 621-631 (1998)

15. Kim, TS, Ko, MH, Choi, YK: The invariance principle for linear multiparameter stochastic processes generated by associated random fields. Stat. Probab. Lett. 78, 3298-3303 (2008) 